\title{
EFFECTIVENESS OF THE REVERSE BENDING AND STRAIGHTENING TESTS IN DETECTING LAMINATIONS IN WIRES FOR CIVIL ENGINEERING APPLICATIONS
}

\author{
K.K. ADEWOLE ${ }^{1}$, S.J. BULL ${ }^{2}$
}

\begin{abstract}
The reverse bending and straightening test is conducted on wires used for civil engineering applications to detect laminations which can pose a threat to the integrity of the wires. The FE simulations of the reverse bending and straightening of wires with laminations revealed that the reverse bending and straightening test is only effective in revealing or detecting near-surface laminations with lengths from $25 \mathrm{~mm}$ located up to $30 \%$ of the wire's thickness and may not be an effective test to detect mid-thickness, near-mid-thickness, and short near-surface laminations with lengths below $15 \mathrm{~mm}$. This is because wires with mid-thickness, near-mid-thickness and short nearsurface laminations will pass through the reverse bending and straightening procedures without fracturing and therefore mid-thickness, near-mid-thickness and short near-surface laminations may go undetected. Consequently, other in-line non destructive testing methods might have to be used to detect mid-thickness, near-mid-thickness and short near-surface laminations in the wires.
\end{abstract}

Key words: FE simulation, Laminations, Reverse bending, Wire

\section{INTRODUCTION}

Carbon steel wires used for civil engineering applications are manufactured by cold rolling or by hot rolling and cold finish rolling processes. For instance, they are used as pre-stressing tendons in pre-stressed concrete structures; provide tensile reinforcement in flexible pipes, and supports for suspension and cable-stayed bridge decks. As part of the quality assurance tests on the wires, they are subjected to a reverse bending and straightening test to detect crack-like laminations that could be inimical to the integrity of the wires in service. A lamination is a material separation or discontinuity in the material which is generally parallel to the rolling or drawing direction SмIтH et al [1]. It is essential to detect laminations in wires as the presence of pre-service crack-like longitudinal laminations in pre-stressing wires has been identified by the United States Bureau of Reclamation [2] as a primary material factor responsible for the catastrophic

1 School of Chemical Engineering and Advanced Materials, Newcastle University, Newcastle upon Tyne, United Kingdom, NE1 7RU, E-mail: kkadewole@yahoo.com, (Corresponding author).

2 School of Chemical Engineering and Advanced Materials, Newcastle University, Newcastle upon Tyne, United Kingdom, NE1 7RU, E-mail: s.j.bull@ncl.ac.uk 
rupture of pre-stressed concrete pipes. Also laminations that are parallel to the longitudinal direction of the wire could provide a good site for hydrogen combination in a hydrogen-containing environment to produce Hydrogen Induced Cracking (HIC) and blistering of wires in service, both of which could threaten the integrity of the wires. The routine reverse bending and straightening test is conducted on carbon steel wires using three rotating rollers in an arrangement shown in Figure 1. Typically, the rollers are $100 \mathrm{~mm}$ in diameter and the wires are bent over the rotating left hand roller, reverse bent over the rotating middle roller and finally straightened over the rotating right hand roller.

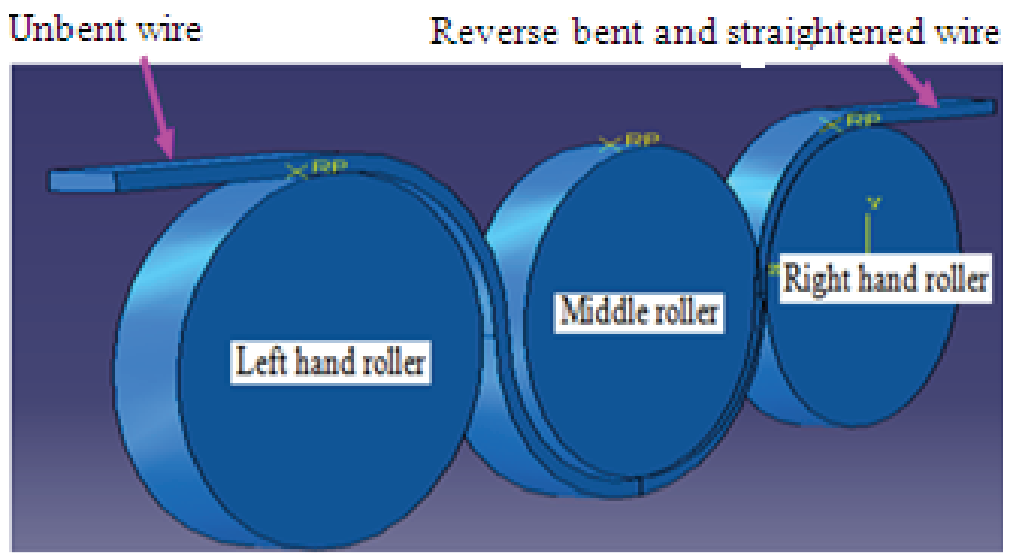

Fig. 1. Industrial reverse bending equipment with three rollers

In this work, an investigation of the effectiveness of the reverse bending and straightening test to detect laminations in typical carbon steel wires for civil engineering applications is presented. The investigation involves laboratory and FE simulations of the reverse bending and straightening (RBS) process. The FE analysis covers simulations of the reverse bending and straightening of lamination free wire specimens and wire specimens with near-surface and mid-thickness laminations.

\section{EXPERIMENTAL}

The reverse bent and straightened tensile test specimens were prepared by winding/ bending a length of the $12 \mathrm{~mm} \times 5 \mathrm{~mm}$ wire round a $100 \mathrm{~mm}$ roller, reverse bending of the bent wire in the opposite direction round the same $100 \mathrm{~mm}$ roller, straightening of the reverse bent wire and cutting of the reverse bent and straightened wire length into tensile test specimens. The unbent tensile test specimens were prepared from the as-received unbent wire length. Details of the test equipment and test conditions at which the laboratory tensile tests were conducted can be obtained from the work of 
Adewole and Bull [3]. The laboratory test was conducted on lamination-free wires as it was not possible to simulate crack-like defects parallel to the length of the wire specimens using machining techniques.

\section{FE ANALYSIS PROCEDURES}

Figure 2a shows the specimen-attachments-rollers-guide plate assembly employed for the simulations of the reverse bending and straightening of the lamination-free wires and the wires with laminations. The lamination is not visible in the meshed image in Figure 2(a) because the lamination was modelled as a seam (a line used in Abaqus for modelling cracks and faces that are originally closed but open during analyses) to simulate the crack-like laminations that may be present in typical carbon steel wires used for civil engineering applications. The lamination is shown in the wireframe model in Figure 2(b). The assembly consists of $100 \mathrm{~mm}$ diameter left (Roller 1) and right rollers (Roller 2), 305mm wire length which consist of a $50 \mathrm{~mm}$ long tensile testing specimen in between $127.5 \mathrm{~mm}$ long left and right attachments, and a $300 \times 20 \times 2 \mathrm{~mm}$ guide plate. The guide plate was introduced to prevent Roller 2 from lifting vertically upward during the bending simulation. The attachments were introduced to prevent localised deformation of the ends of the tensile testing specimen, which occurred when the simulation was conducted with the specimen bonded directly to the rollers. The left roller (Roller 1) and the left attachment were modeled together as a unit as the reverse bending and straightening process simulations conducted with the roller and the attachment modeled as two separate units with the attachment bonded to the roller could not be completed. This was because the attachment broke off from the roller at the location of the bond between the roller and the attachment during the reverse bending simulation. Similarly, the right roller (Roller 2) and the right attachment were modeled together as a unit. The $50 \mathrm{~mm}$ test specimen was also modeled as a unit and all the nodes on its left and right ends were bonded to the nodes at the end of the left and right attachments respectively.

The whole model was meshed with C3D8R elements (8-node hexahedral linear brick reduced integration elements with hourglass control). Each roller was meshed with a total of four thousands, seven hundreds and seventy two $3 \times 3 \times 3 \mathrm{~mm}$ elements and the guide plate was meshed with seven hundred $3 \times 3 \times 3 \mathrm{~mm}$ elements. Each attachment and the specimen were meshed with one thousand nine hundred and twenty $3 \times 3 \times 0.5 \mathrm{~mm}$ and two thousands $3 \times 1 \times 0.5 \mathrm{~mm}$ elements respectively. The $1 \mathrm{~mm}$ dimension is along the specimen length and the $0.5 \mathrm{~mm}$ dimension is along the specimen thickness, which translates to 10 elements along the wire thickness. The $50 \mathrm{~mm}$ long specimen which was employed to study the stresses, the strains and the deformation behaviors of the lamination free-wire and the wire with laminations during the bending, reverse bending and straightening simulations was meshed with the finest mesh size $(3 \times 1 \times 0.5 \mathrm{~mm})$ while the rollers, the guide plate and the attachments were meshed with 


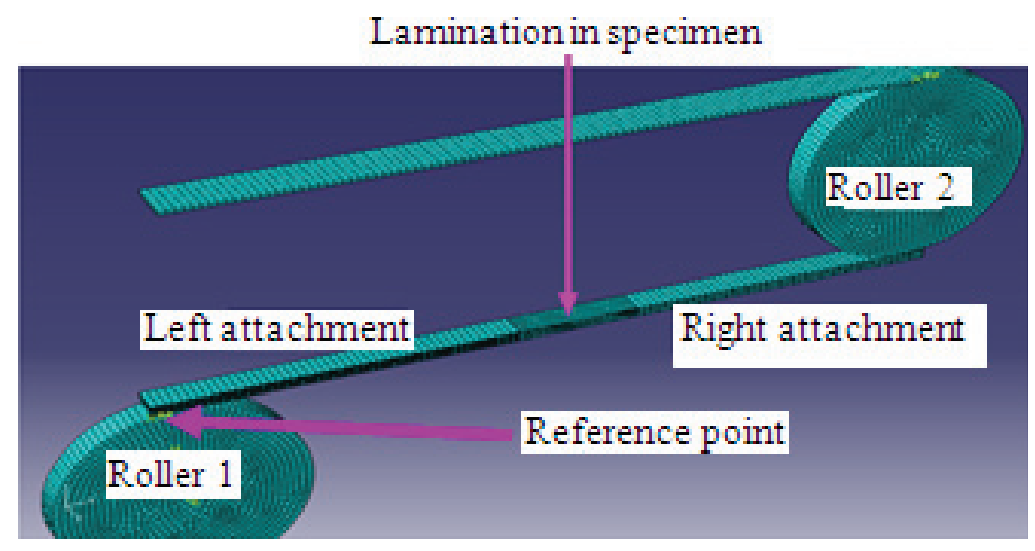

(a) Meshed specimen-rollers-attachments assembly

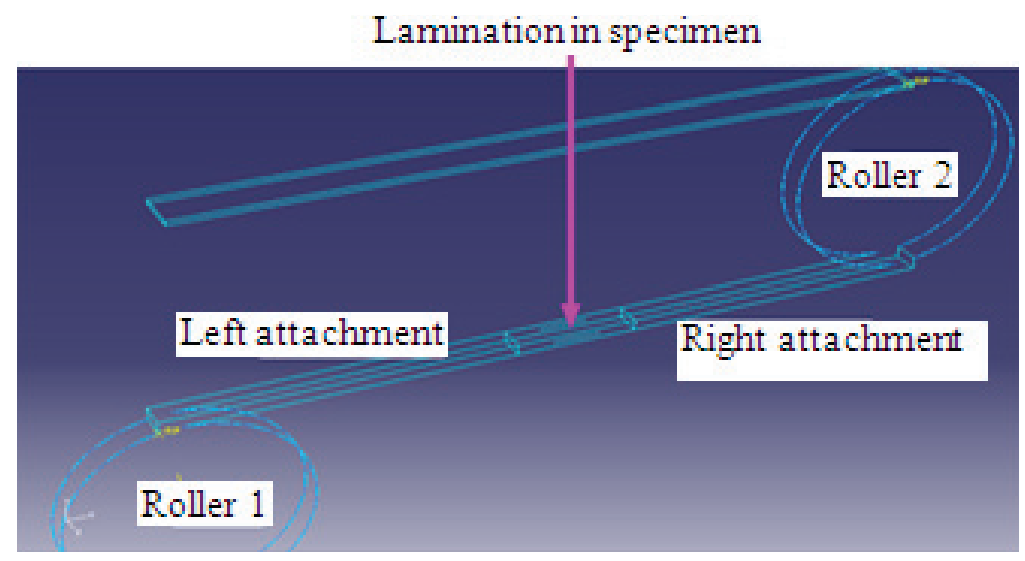

(b) Assembly of specimen-rollers-attachments in wire

Fig. 2. Specimen with lamination in specimen-rollers-attachments assembly

a coarse mesh to reduce the output file size and computation time. The $3 \times 1 \times 0.5 \mathrm{~mm}$ element size was established through mesh convergence studies to be the optimum mesh size for an accurate prediction of the responses of the wire throughout the bending, reverse bending and straightening simulations.

\subsection{Material constitutive models and modeling PaRameters}

The simulations of the reverse bending and straightening of lamination-free wire and that of the wire with laminations were conducted using the combined hardening model in combination with the phenomenological shear damage and failure model in-built in the Abaqus v 6.9.3 material library. The details of the combined hardening model, the 
phenomenological shear damage and failure model and their modeling parameters have been published by Adewole [4,5], and Adewole and Bull [6] respectively.

\subsection{FE SIMULATION PROCEDURES}

The FE simulations of the bending, reverse bending, straightening and tensile testing of the lamination-free wire specimen and the wire specimens with $5 \mathrm{~mm}, 10 \mathrm{~mm}, 15 \mathrm{~mm}$, $20 \mathrm{~mm}, 25 \mathrm{~mm}$ and $30 \mathrm{~mm}$ laminations located at $0.5 \mathrm{~mm}, 1.0 \mathrm{~mm}, 1.5 \mathrm{~mm}, 2.0 \mathrm{~mm}$, and $2.5 \mathrm{~mm}$ below the top surface of the flat wire were conducted in four simulation steps using the specimen-attachments-rollers-guide plate assembly shown in Figure 2a. The laminations located at $0.5 \mathrm{~mm}$ and $1.0 \mathrm{~mm}, 1.5 \mathrm{~mm}$ and $2.0 \mathrm{~mm}$, and $2.5 \mathrm{~mm}$ below the top surface of the wire are hereinafter referred to as near-surface, near-mid-thickness and mid-thickness laminations respectively. The wire's bending simulation was conducted during the first simulation step by subjecting Roller 1's reference point to an angular velocity of 10 radians/second in the Z-axis direction which rotated Roller 1 in an anticlockwise direction round the Z-axis and bent/wound the wire round Roller 1. The reverse bending simulation was conducted during the second simulation step by rotating Roller 1 in a clockwise direction to unwind the wire from Roller 1 whilst simultaneously rotating Roller 2 in an anticlockwise direction to reverse bend and wind the wire round Roller 2. Roller 1 was rotated in a clockwise direction by subjecting its reference point to an angular velocity of -10 radians/second in the Z-axis direction. Roller 2 was rotated in an anticlockwise direction by subjecting its reference point to an angular velocity of 10 radians/second in the Z-axis direction. The simulation of the straightening of the reverse bent wire was conducted during the third simulation step by rotating Roller 2 in a clockwise direction to unwind the wire whilst simultaneously pulling Roller 1 longitudinally through a distance of $187.5 \mathrm{~mm}$ in the X-axis direction. Roller 2 was rotated in a clockwise direction by subjecting its reference point to an angular velocity of -1.375 radians/second in the $\mathrm{Z}$-axis direction. The element removal option of the shear failure model was employed by using the default value of 1 as the damage variable to simulate fracture in the wire by element removal.

The simulations of the tensile testing of the numerically reverse bent and straightened wire specimen was conducted during the fourth simulation step by fixing the left hand end of the specimen, the left roller (Roller 1) and the left attachment and subjecting the right hand end of the specimen, the right roller (Roller 2) and the right attachment, which were free to move only in the tensile load direction to a longitudinal axial tensile displacement. The simulation of the tensile testing of the unbent wire specimen was conducted with the same specimen-attachments-rollers-guide plate assembly and the same boundary conditions employed for the simulation of tensile testing of the reverse bent and straightened wire specimen to ensure that the same simulation procedure was employed for the tensile testing of the unbent and the reverse bent and straightened wire specimens. However, the simulation of the tensile testing of the unbent wire specimen was conducted with the bending, reverse bending and straightening simulations steps 
suppressed (i.e. made inactive). Effectively, the tensile testing simulation step was the only active step during the simulation of the tensile testing of the unbent wire specimen.

\section{RESULts}

The deformed shapes of the specimens with the near-mid-thickness and mid-thickness laminations during the bending and reverse bending simulations are the same and only the deformed shapes of the specimens with the mid-thickness laminations are presented. The deformed shape of the whole $305 \mathrm{~mm}$ wire length and that of the $50 \mathrm{~mm}$ long lamination free wire specimen and the wire specimens with $5 \mathrm{~mm}, 10 \mathrm{~mm}, 15 \mathrm{~mm}$, $20 \mathrm{~mm}, 25 \mathrm{~mm}$ and $30 \mathrm{~mm}$ near-surface and mid-thickness laminations after the bending simulation are the same and only the deformeds shape of the specimen with $20 \mathrm{~mm}$ long laminations are presented in Figure 3. The lamination in the bent $50 \mathrm{~mm}$ wire specimen is not visible in the meshed image in Figure 3(a) and is as shown in the wireframe image in Figure 3(b). The through-thickness longitudinal stress and equivalent plastic strain (PEEQ) distributions in the bent $50 \mathrm{~mm}$ wire specimen with $20 \mathrm{~mm}$ long lamination are shown in Figures 3(c) and (d) respectively. In the longitudinal axial stress contours, tensile stresses are positive with the maximum tensile stress represented by the deepest red colour, while compressive stresses are negative with the maximum compressive stress represented by the deepest blue colour.

The deformed shapes of the whole $305 \mathrm{~mm}$ wire length and the $50 \mathrm{~mm}$ test specimen showing the longitudinal axial stress distribution in the model of the lamination-free wire (a), wire with the near-surface lamination (b) and the wire with the mid-thickness lamination (c) during and after the reverse bending simulations are shown in Figures 4 and 5 respectively. The deformed shapes of the whole $305 \mathrm{~mm}$ wire length and the $50 \mathrm{~mm}$ test specimen showing the longitudinal axial stress distribution in the wire model without the lamination defect, with the near-surface lamination and with the mid-thickness lamination during and after the straightening simulations are shown in Figures 6 (a), (b) and (c) and Figures 7 (a), (b) and (c) respectively. The deformed shapes of the $50 \mathrm{~mm}$ specimens with sections of the left and right attachments showing the lamination-free wire specimen and the wire specimens with varying lamination lengths and locations after the straightening simulation are shown in Figures 8(a) to (g). From Figures $8(\mathrm{a})$ to $(\mathrm{g})$, the specimens with varying lamination lengths and at varying lamination locations exhibit there distinct deformed or fracture shapes with completely fractured thin ligament (8e), partially fractured thin ligaments (8d) and unfractured or unbroken ligaments $(8 \mathrm{a}-\mathrm{c})$. The details of the deformed or fracture shapes exhibited by the $5 \mathrm{~mm}, 10 \mathrm{~mm}, 15 \mathrm{~mm}, 20 \mathrm{~mm}, 25 \mathrm{~mm}$ and $30 \mathrm{~mm}$ laminations located at $0.5 \mathrm{~mm}$, $1.0 \mathrm{~mm}, 1.5 \mathrm{~mm}, 2.0 \mathrm{~mm}$, and $2.5 \mathrm{~mm}$ below the top surface of the flat wire after the reverse bending and straightening simulations are presented in Table 1. The normalised force-displacement curves obtained from the experimental and FE tensile testing of the 
unbent and the reverse bent and straightened wire specimens are shown in Figures 9(a) and (b) respectively.

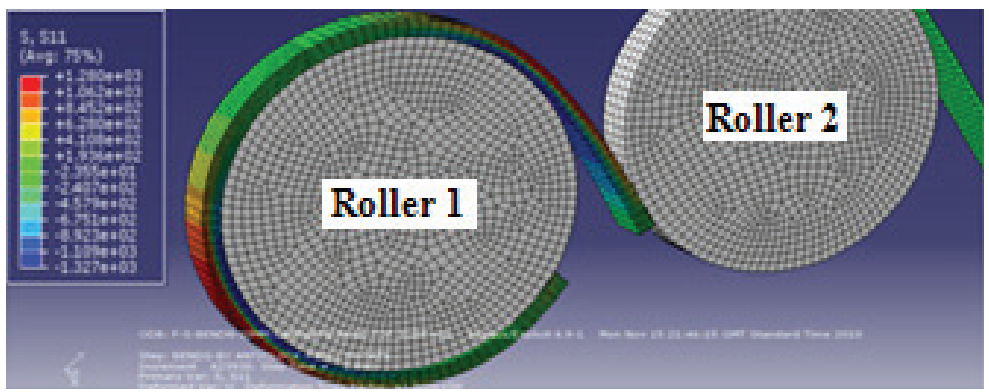

(a) Meshed image of wire with lamination bent round roller

\section{Lamination in specimen}

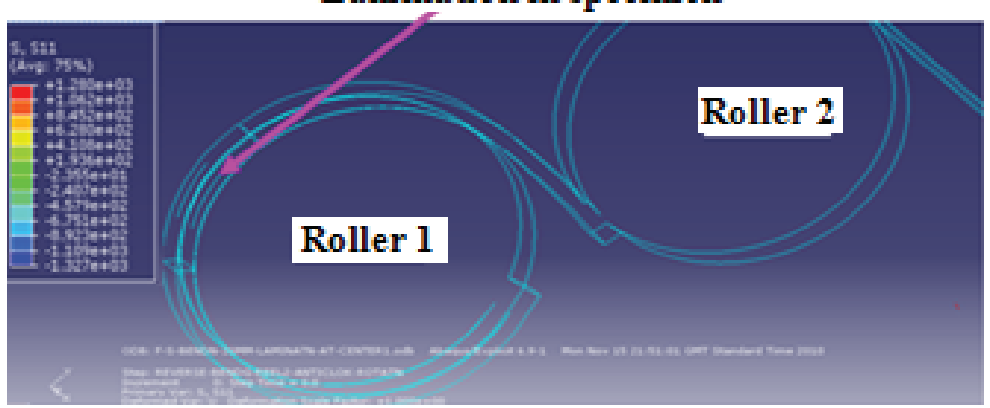

(b) Wire framed image of wire with lamination bent round roller.

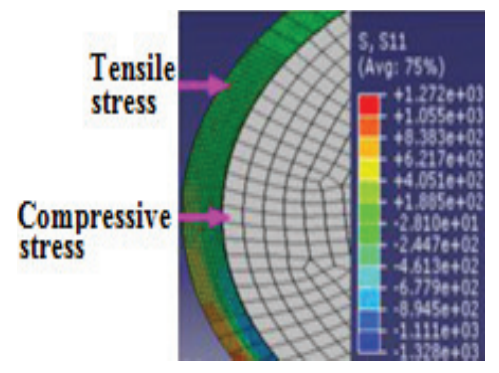

(c) Longitudinal stress

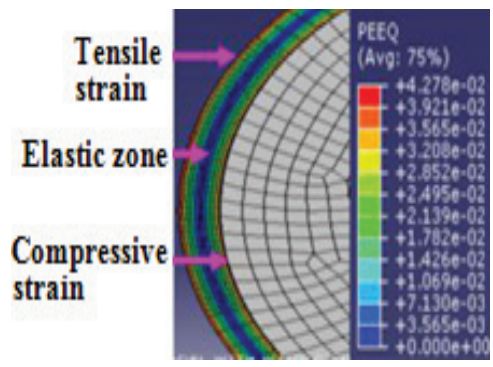

(d) Equivalent plastic strain

Fig. 3. Deformed shapes with longitudinal stress (MPa) and equivalent plastic strain distributions in wire specimens with $20 \mathrm{~mm}$ long lamination after bending simulation 


\section{VALIDATION AND VERIFICATION OF FE SIMULATION RESULTS}

The details of the verification and validation procedures have been published by AdEwole [7] and only the summary of the procedures are presented. The simulation of the reverse bending and straightening of wires with laminations could not be validated with experimental results as it was not experimentally possible to machine line-type or crack-like defects that are parallel to the length of the wire specimens into the experimental wire specimens using machining techniques. Consequently, only the verification of the reverse bending and straightening simulations are presented. Figure 3 shows that the bending process subjects the upper part of the wire to a tensile stress and the lower part of the wire to a compressive stress which agrees with the stress pattern in a bent wire stated by GILLSTROM and JARL [8] and demonstrates the accuracy of the bending simulation. The bending simulation predicted peak stress and strain at the surfaces of the wire specimen, which agrees with what TVERGAARD [9] (1987) reported and further demonstrates the reliability of the bending simulation procedure. Using the expression for the maximum strain (e) at the top surface of a bent wire in equation 5.1 obtained from Gillstrom and JARL [8], the maximum strain in the bent wire with thickness (T) of $5 \mathrm{~mm}$, bent round a roller with diameter (D) of $100 \mathrm{~mm}$ is 0.048 which agrees with the maximum strain value of 0.043 predicted by the bending simulation as shown in Figure 3(d). The good agreement between the maximum strain values obtained from the FE simulation and the analytical expression further demonstrates the accuracy of the bending simulation. The good agreements between the experimental and FE force-displacement curves for the tensile testing of the unbent and reverse bent and straightened wire specimens shown in Figures 9(a) and (b) respectively demonstrates the accuracy of the bending, reverse bending, straightening and tensile testing simulations. It demonstrates that the simulation methodology employed for the simulation of the bending, reverse bending, straightening and tensile testing of the numerically reverse bent and straightened wire are able to predict the behavior of wires subjected to the reverse bending and straightening process. The verification of the straightening process simulation was conducted by comparing the stress distribution and the strain profiles predicted by the simulation with the expected stress and strain profiles of a wire subjected to reverse bending and straightening. Details have been published by Adewole, [4]. Having verified the accuracy of the FE simulation of the RBS processes, the same simulation procedures were employed for the simulation of the RBS of wire specimens with laminations.

$$
e=\frac{T}{T+D}=\frac{5}{5+100}=0.048
$$




\section{Discussion}

As shown in Figure 3, the bending process subjects the upper part of the wire to a tensile stress and the lower part of the wire to a compressive stress. There was no noticeable difference in the deformed shape of the wire either with or without laminations after the bending simulation. From Figure 4, it can be seen that during the reverse bending simulation which subjected the upper and the lower part of the wire to compressive and tensile stresses respectively, the compressive stress in the upper ligaments of the wires containing laminations caused the ligaments to buckle, thereby creating an opening. The opening was larger in the specimen with the near surface lamination shown in Figure 4(b) as the thin ligament buckled more than the ligament of the specimen with the midthickness lamination shown in Figure 4(c). No opening was observed in the lamination free wire as shown in Figure 4(a). At the end of the reverse bending simulation, the opening in the specimen with the near-surface lamination remained, as shown in Figure 5(b), because the roller was unable to flatten out the buckled thin ligament due to its high degree of curvature. The opening in the specimen with the mid-thickness lamination closed up as shown in 5(c) because the buckled ligament was flattened by the roller due to its gentle curvature. No opening was observed in the lamination free wire specimen after the reverse bending simulation as shown in Figure 5(a).

During the straightening simulation, the upper and lower parts of the wire specimen were subjected to compressive and tensile stresses respectively as shown in Figure 6. The tensile stress at the lower part of the wire continuously straightened the curved thin ligament of the specimen with the near-surface lamination. The application of the tensile stress to the thin ligament increased the tensile strain in the ligament and eventually led to fracture initiation in the thin ligament typified by element removal as shown in Figure $7\left(b_{1}\right)$ when the equivalent plastic strain in the thin ligament reached the specified 0.44176 fracture strain. At the end of the straightening simulation, the thin ligament completely fractured as shown by the removal of a row of elements across the entire thickness of the thin ligament in Figure $7\left(b_{2}\right)$. At the beginning of the straightening simulation, the ligament above the mid-thickness lamination buckled under the applied compressive stresses, leading to an opening up of the specimen at the location of the mid-thickness lamination as shown in Figure 7(c). Towards the end of the straightening simulation, the stress across the entire wire thickness became tensile and at the end of the straightening simulation, the opening at the location of the mid-thickness lamination had partially closed up as shown in Figure 7(c) due to the straightening of the buckled ligament by the tensile stress. No fracture of the mid-thickness lamination was observed after the straightening simulation. Neither opening nor partial fracture was observed on the lamination- free wire specimen after the straightening simulation as shown in Figure $7(\mathrm{a})$. 


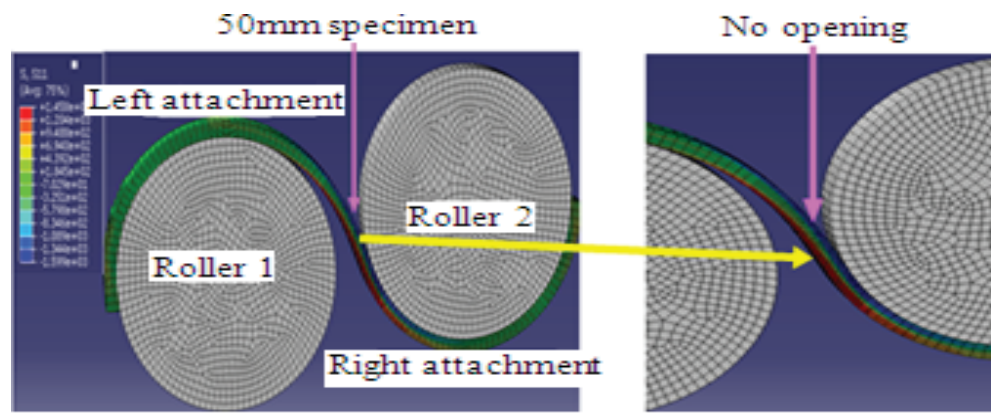

(a) Lamination free wire
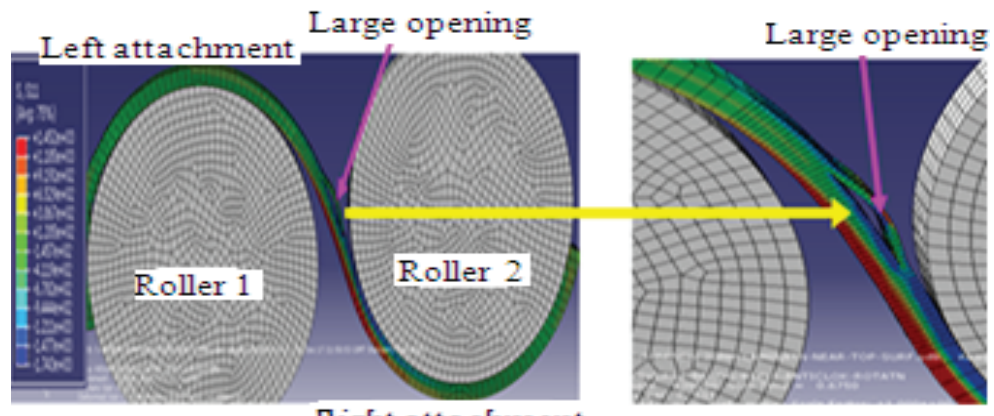

Right attachment

(b) Wire with near-surface lamination

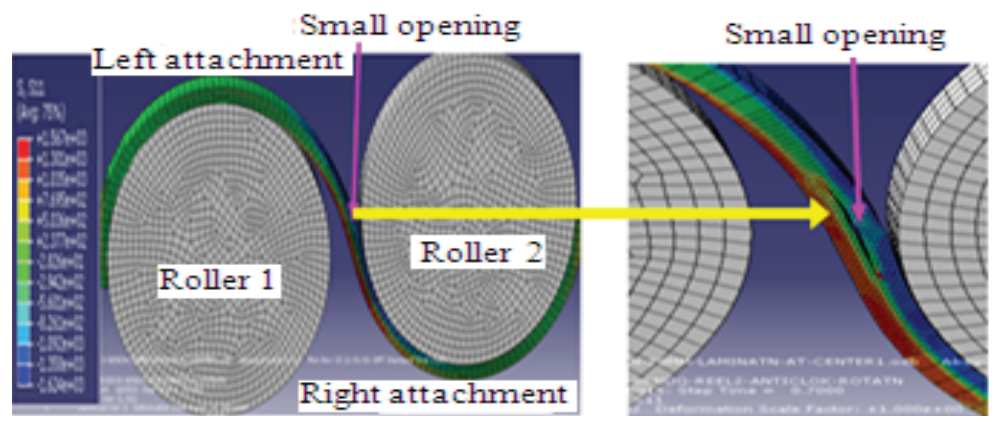

(c) Wire with mid-thickness lamination

Fig. 4. Deformed shapes of the whole $305 \mathrm{~mm}$ wire length and the $50 \mathrm{~mm}$ test specimen with and without $20 \mathrm{~mm}$ long laminations during reverse bending simulation 


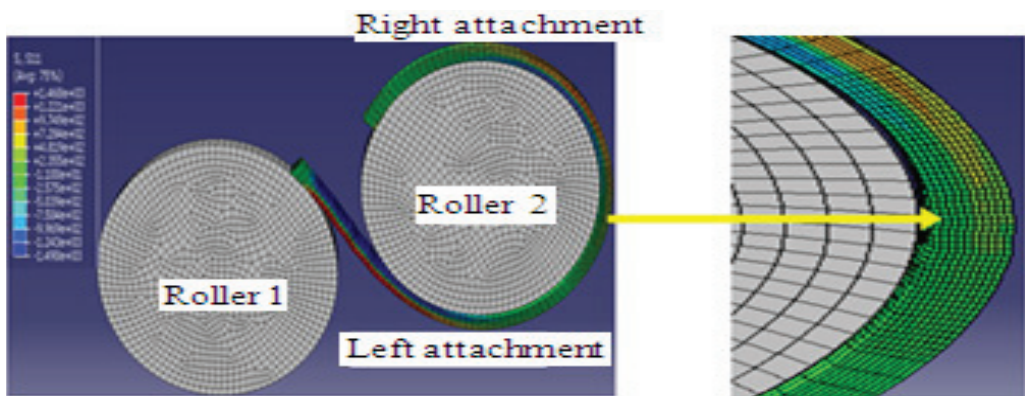

(a) Lamination free wire

\section{Large opening}

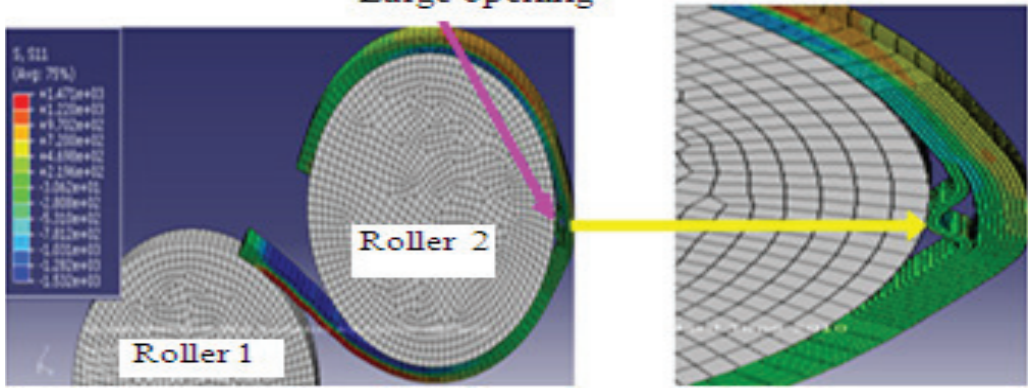

(b) Wire with near-surface lamination

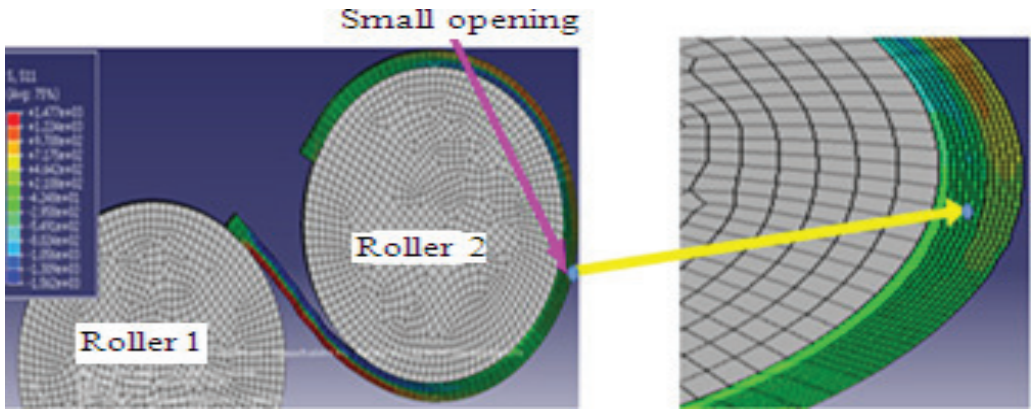

(c) Wire with mid-thickness lamination

Fig. 5. Deformed shapes of the whole $305 \mathrm{~mm}$ wire length and the $50 \mathrm{~mm}$ test specimen with and without $20 \mathrm{~mm}$ long laminations after reverse bending 


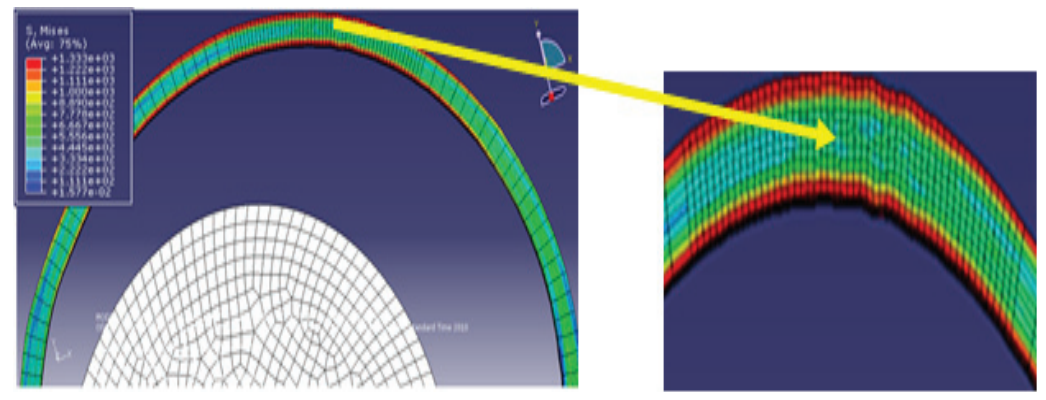

(a) Lamination free wire

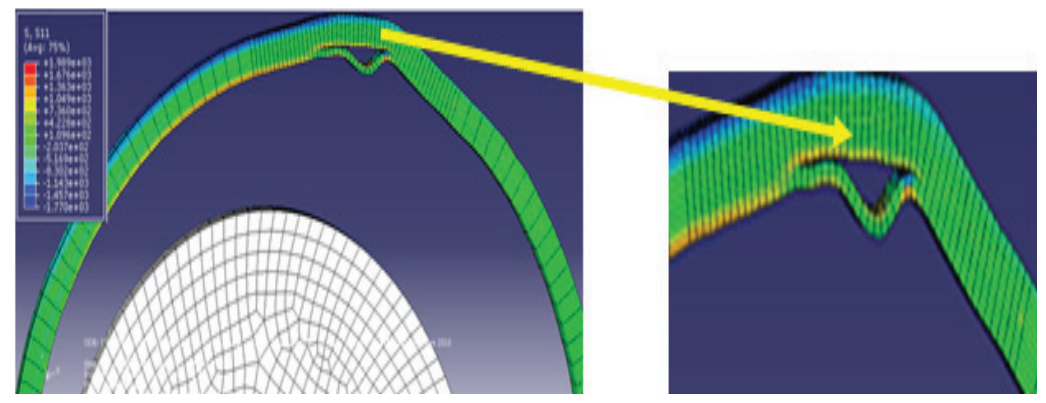

(b) Wire with near-surface lamination

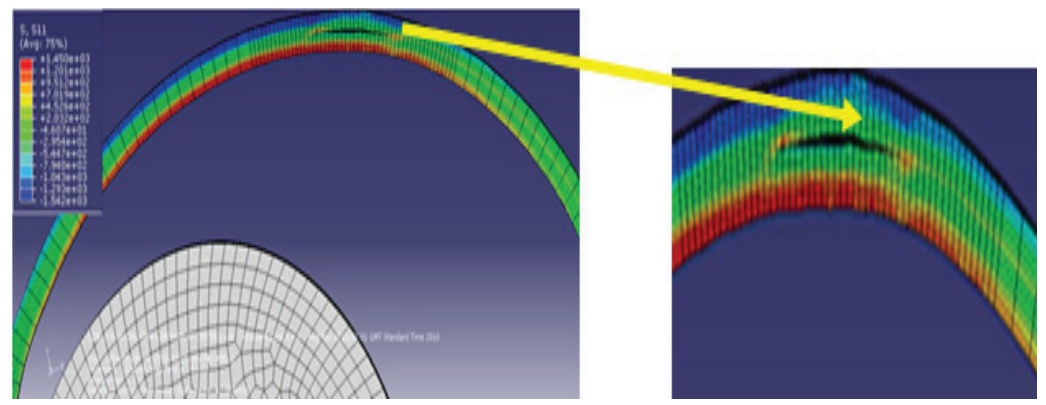

(c) Wire with mid-thickness lamination

Fig. 6. Deformed shapes of the whole $305 \mathrm{~mm}$ wire length and the $50 \mathrm{~mm}$ test specimen with and without $20 \mathrm{~mm}$ long laminations during the straightening simulation 


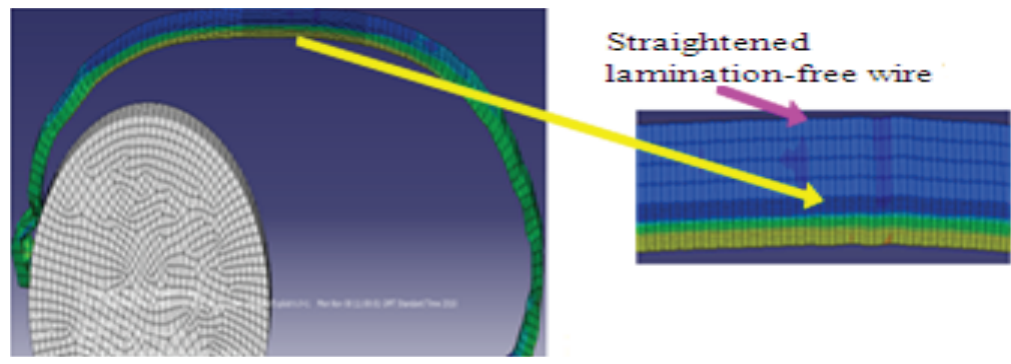

(a) Lamination free wire

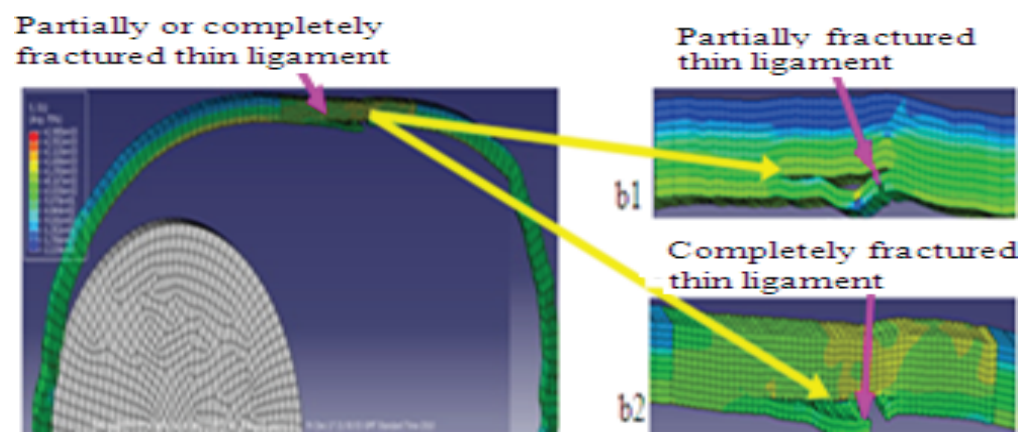

(b) Wire with near-surface lamination

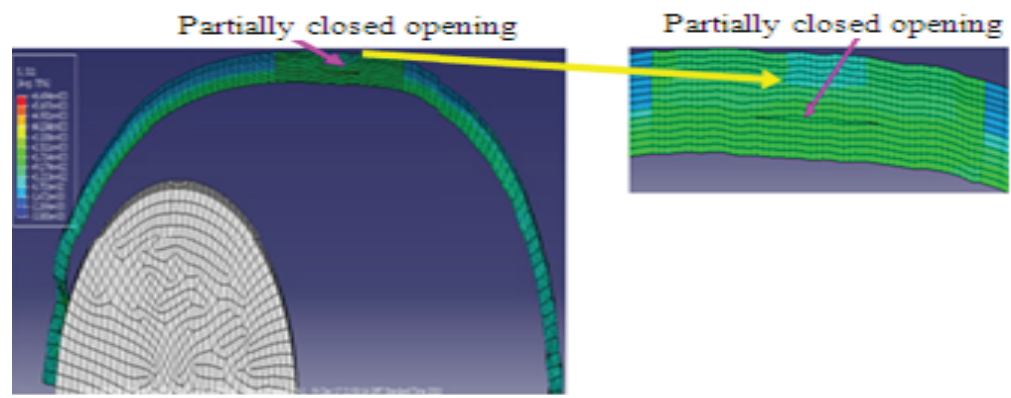

(c) Wire with mid-thickness lamination

Fig. 7. Deformed shapes of the whole $305 \mathrm{~mm}$ wire length and the $50 \mathrm{~mm}$ test specimen with and without $20 \mathrm{~mm}$ long laminations after the straightening simulation 
Depending on the length and location of the laminations, the thin ligament of the wire specimens with the near-surface and the near-mid-thickness laminations completely fractured, partially fractured, or remained unfractured as shown in Figures (8e), (8d) and $8(\mathrm{c})$ respectively. No fracture was observed in any of the two equal ligaments of the wire specimens with mid-thickness laminations for all the lamination lengths and locations considered. The laminations in wire specimens that fractured partially were widened by the reverse bending and straightening process as the originally line-type laminations that is not visible in the meshed model in Figure 2 is now slightly visible in the reverse bent and straightened wire specimens as shown in Figures 8(b), (c), (f) and (h). Although the widened laminations are slightly visible in the deformed FE model of the wire, they may not be visible to the naked eyes.

From Table 1, the status (completely fractured, partially fractured or "unfractured") of the reverse bent and straightened wire specimens with laminations depends on the combination of the lamination length and location. Generally, the longer the lamination and the nearer to the surface of the wire the lamination is, the more likely the thin ligament fractures. This is due to the fact that the longer the lamination, the more severe the buckling and the bending stress/strain the thin ligament experiences during the reverse bending operation. Similarly, the nearer to the surface of the wire the lamination is located, the thinner the thin ligament and the more severe the buckling and the bending stress/strain the thin ligament experiences. Consequently, the thin ligaments of the long (lengths greater than or equal to $25 \mathrm{~mm}$ ) near-surface laminations and the long near-mid-thickness laminations have been subjected to a high plastic deformation when compared with the thin ligaments of the short (lengths less than or equal to $10 \mathrm{~mm}$ ) nearsurface laminations, the thin ligaments of the short near-mid-thickness laminations and the thin ligaments of the short, long and medium length (lengths greater than $10 \mathrm{~mm}$ and less than $25 \mathrm{~mm}$ ) mid-thickness laminations.

Table 1

Status of lamination at the end of reverse bending and straightening process

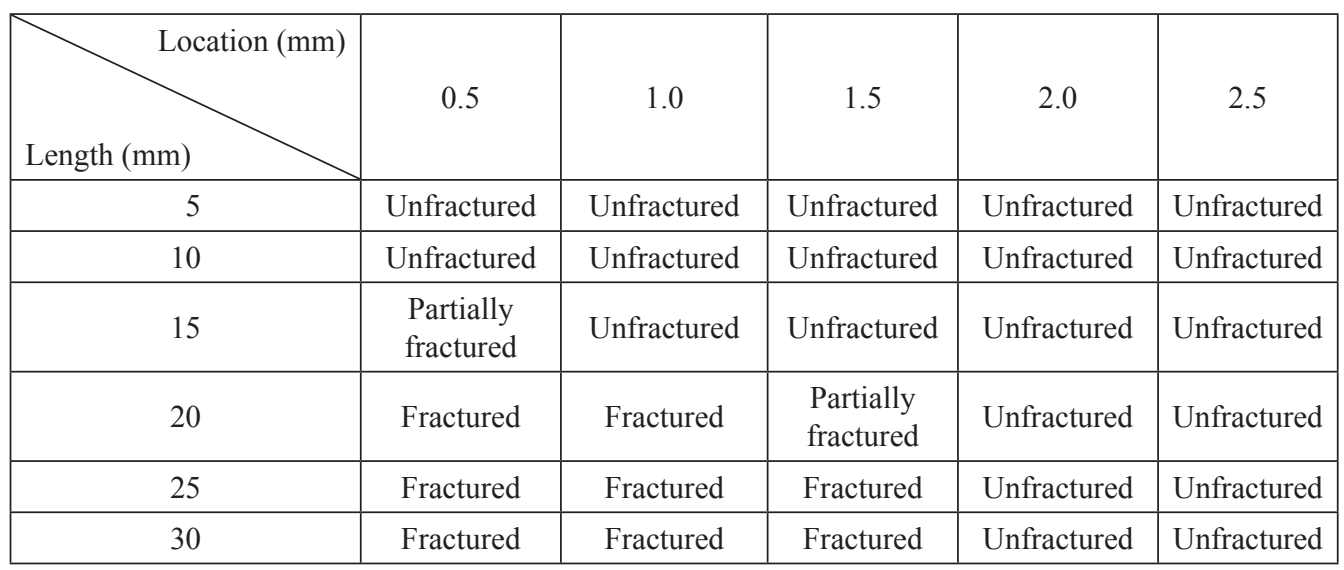




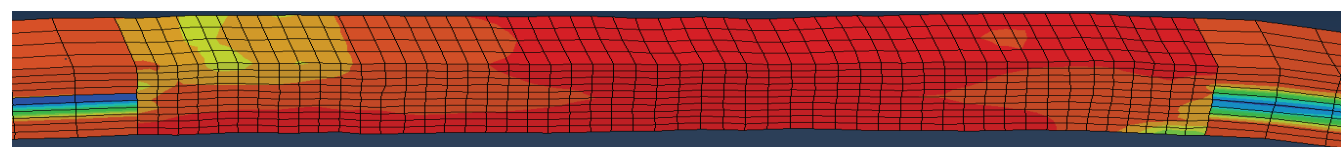

a) Lamination-free wire

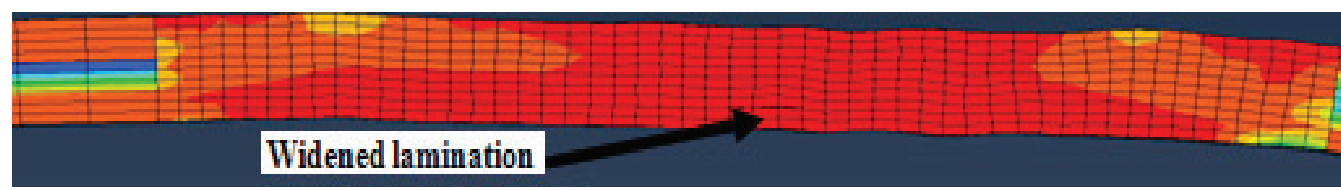

(b) Wire specimen with $5 \mathrm{~mm}$ lamination located $1.0 \mathrm{~mm}$ below wire's top surface

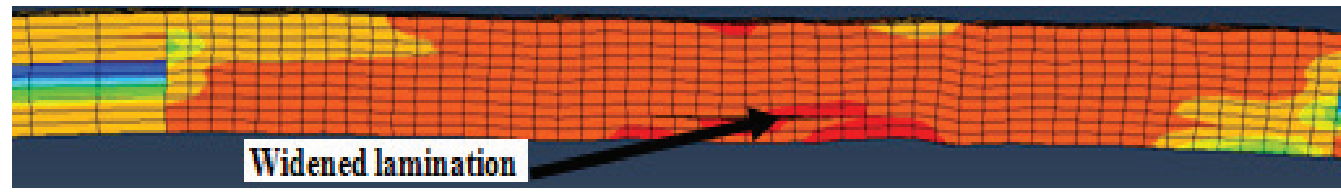

(c) Wire specimen with $10 \mathrm{~mm}$ lamination located1.0mm below wire's top surface

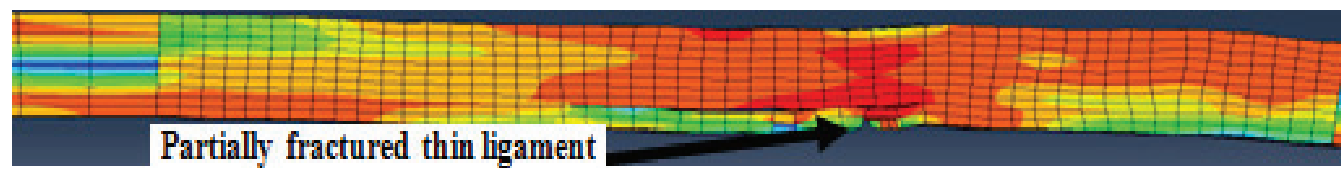

(d) Wire specimen with $15 \mathrm{~mm}$ lamination located $1.0 \mathrm{~mm}$ below wire's top surface

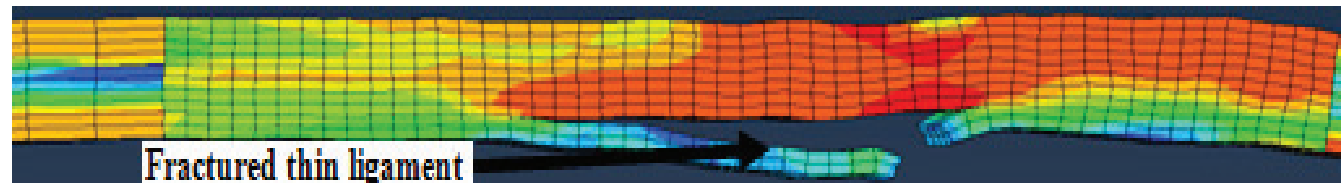

(e) Wire specimen with $20 \mathrm{~mm}$ lamination located $1.0 \mathrm{~mm}$ below wire's top surface

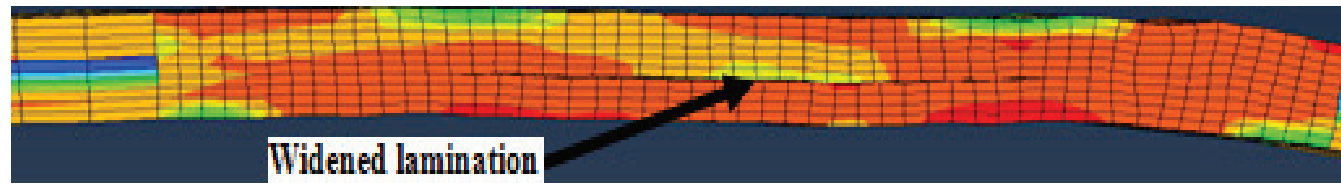

(f) Wire specimen with $25 \mathrm{~mm}$ lamination located $2.0 \mathrm{~mm}$ below top surface

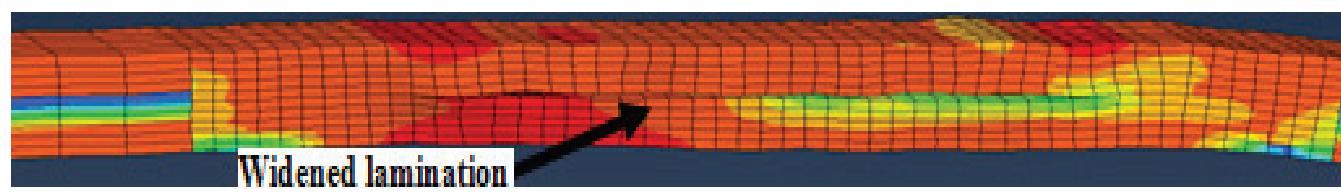

(g) Wire specimen with $30 \mathrm{~mm}$ lamination located $2.5 \mathrm{~mm}$ below wire's top surface

Fig. 8. Deformed shapes of wire specimens with varying lamination lengths and locations after the straightening simulation 
The high plastic deformation experienced by the long thin ligaments has reduced the total amount of available plastic straining the thin ligaments could undergo before fracturing. This explains why the thin ligaments of the long near-surface laminations and the thin ligaments of the long near-mid-thickness laminations fractured or partially fractured under the same tensile stress/strain induced by the straightening process which did not cause the fracture of the thin ligaments of the wire specimens with short nearmid-thickness, and the wire specimens with the short, long and medium length midthickness laminations. Specifically, the differences in the amount of available plastic straining the thin ligament could undergo before fracturing explains why the specimens with long near-surface laminations located up to $1.5 \mathrm{~mm}$ ( $30 \%$ of wire's depth) below the wire's surface fractured while the specimens with short near-surface laminations located at all the depths considered (up to $50 \%$ of wire's thickness) did not fracture. This also explains why the wire specimen with medium length near-surface laminations and medium length near-mid-thickness laminations located at $1.5 \mathrm{~mm}(30 \%$ of the wire's thickness) completely fractured, partially fractured or unfratured. Similarly, for all the lamination lengths (long, short and medium) considered, the wire specimens with near-mid-thickness laminations located at $2.0 \mathrm{~mm}$ ( $40 \%$ of the wire's thickness) and the wire specimens with mid-thickness laminations located at all the depths considered did not fracture.

\section{Conclusion}

The investigation of the effectiveness of the reverse bending and straightening operations in detecting laminations through the finite element simulations shows that the effectiveness of the reverse bending and straightening operations in detecting laminations in typical carbon steel wires used for civil engineering application depends on the length and location of the lamination. The reverse bending and straightening test is only effective in revealing long near-surface laminations with lengths greater than or equal to $25 \mathrm{~mm}$ located up to $1.5 \mathrm{~mm}$ ( $30 \%$ of the wire's depth) below the wire's surface. The reverse bending and straightening test may not be an effective test in detecting mid-thickness laminations with length up to $30 \mathrm{~mm}$, near-mid-thickness laminations with lengths greater than $10 \mathrm{~mm}$ and less than $25 \mathrm{~mm}$ located $2 \mathrm{~mm}$ or more below the wire's surface and short near-surface laminations with lengths less than or equal to $10 \mathrm{~mm}$ located at all depths up to $2.5 \mathrm{~mm}(50 \%$ or middle of the wire's thickness) below the wire's surface. This is because wires with long near-surface laminations can readily be detected visually as the thin ligament breaks during the straightening operation. However, wires with short near-surface laminations, midthickness laminations with length up to $30 \mathrm{~mm}$ and near-mid-thickness laminations with lengths greater than $10 \mathrm{~mm}$ and less than $25 \mathrm{~mm}$ located $2 \mathrm{~mm}$ or more below the wire's surface could pass through the reverse bending and straightening procedures without fracturing. Although these seemingly harmless line type laminations are 
widened by the reverse bending and straightening process they may go undetected by the naked eye.

As it has been shown in pipeline that harmless parallel mid wall laminations can develop to a critical defects with cavities during service that provide ideal sites for hydrogen recombination mechanisms in a wet $\mathrm{H}_{2} \mathrm{~S}$ (hydrogen charging) environment, the reverse bending and straightening of wires with parallel mid wall laminations, may also leave the wires with pre-service openings that can serve as sites for hydrogen recombination mechanisms, particularly where the wires are used as tensile armour wires in flexible pipes operated in a wet $\mathrm{H}_{2} \mathrm{~S}$ and other hydrogen charging environments, which could lead to blistering and cracking of the wires. Consequently, other in-line non destructive testing methods might have to be used to detect mid-thickness laminations, near-mid-thickness laminations with lengths greater than $10 \mathrm{~mm}$ and less than $25 \mathrm{~mm}$ located $2 \mathrm{~mm}$ or more below the wire's surface and short near-surface laminations in carbon steel wires used for civil engineering applications when used as reinforcement in pre-stressed concrete pipes, flexible pipes and other structures that are operated in hydrogen-containing (hydrogen charging) environments.

\section{REFERENCES}

1. B.O Smith, A.P.H. Jenning, A.G Grimshaw. A portable lamination detector for steel sheet, The British Iron and Steel Research Association, Battersea Park Road, London, 1957.

2. United States Bureau of Reclamation. Prestressed Concrete Pipe Failure Jordan Aqueduct, Reach 3". All U.S. Government Documents (Utah Regional Depository) 1994. Paper 284. http://digitalcommons. usu.edu/govdocs/284.

3. K. K Adewole. Finite Element Simulation of the Reverse Bending and Straightening of Steel Bars Used For Civil Engineering Applications. Global Journal of Researches in Engineering: Civil And Structural Engineering, 12, 4, 1.0, 1-10, 2012.

4. K. K Adewole. Determination of the Appropriate Plasticity Hardening Model for the Simulation of the Reverse Bending and Straightening of Wires for Civil Engineering Applications. Global Journal of Researches in Engineering: Civil And Structural Engineering, 13, 3, 1.0, 43-53, 2013.

5. K. K. Adewole. Identification of Appropriate Micromechanical Fracture Model for Predicting Fracture Performance of Steel Wires for Civil Engineering Applications. Global Journal of Researches in Engineering: Civil And Structural Engineering, 13, 3, 1.0, 24-33, 2013.

6. K. K. Adewole, S. J Bull. Prediction of the fracture performance of defect-free steel bars for civil engineering applications using finite element simulation. Construction and Building Materials, 41, 9-14, 2013.

7. K. K. Adewole. Finite Element Simulation of the Reverse Bending and Straightening of Steel Bars Used For Civil Engineering Applications. Global Journal of Researches in Engineering: Civil And Structural Engineering, 12, 4, 1.0, 1-10, 2012.

8. P. Gillstrom, M. Jarl. Mechanical descaling of wire rod using reverse bending and brushing, Journal of Material Processing Technology, 172, 3, 332-334, 2006.

9. V. TVergandD. Ductile shear failure at the surface of a bent specimen. Mech. Mater., 6, 53-69, 1987. 